\title{
On Potential Equations of Finite Games *
}

\author{
Xinyun Liu, Jiandong Zhu \\ School of Mathematical Sciences, Nanjing Normal University, Nanjing, 210023, P. R. China
}

\begin{abstract}
In this paper, some new criteria for detecting whether a finite game is potential are proposed by solving potential equations. The verification equations with the minimal number for checking a potential game are obtained for the first time. Some connections between the potential equations and the existing characterizations of potential games are established. It is revealed that a finite game is potential if and only if its every bi-matrix sub-game is potential.
\end{abstract}

Key words: Finite game, Potential game, Potential equation, Semi-tensor product.

\section{Introduction}

Game theory, the science of strategic decision making pioneered by John von Neumann (see von Neumann, J. \& Morgenstern, O., 1953), has wide real-world applications in many fields, including economics, biology, computer science and engineering. The Nash equilibrium, named after John Forbes Nash, Jr, is a fundamental concept in game theory. The existence and computing of Nash equilibria are two central problems in the theory of games. For two-player zero-sum games, von Neumann proved the existence of mixed-strategy equilibria using Brouwer Fixed Point Theorem. Nash proved that if mixed strategies are allowed, then every game with a finite number of players and strategies has at least one Nash equilibrium (Nash, 1951). Although pure strategies are conceptually simpler than mixed strategies, it is usually difficult to guarantee the existence of a pure-strategy equilibrium. However, it is shown that every finite potential game possesses a pure-strategy Nash equilibrium (Monderer \& Shapley, 1996). The concept of potential game was proposed by Rosenthal(1973). A game is said to be a potential game if it admits a potential function. The incentive of all players to change their strategy can be expressed by the difference in values of the potential function. For a potential game, the set of pure-strategy Nash equilib-

\footnotetext{
* This work is supported in part by National Natural Science Foundation (NNSF) of China under Grant 11271194 and a project funded by the Priority Academic Program Development of Jiangsu Higher Education Institutions (PAPD). This paper was not presented at any IFAC meeting. Corresponding author J. Zhu. Tel. +86-13851781823.

Email addresses: liuxinyun1224@163.com (Xinyun Liu), jiandongzhu@njnu.edu.cn (Jiandong Zhu).
}

ria can be found by searching the maximal values of the potential function.

An important problem is how to check whether a game is a potential game. Monderer and Shapley (1996) first proposed necessary and sufficient conditions for potential games. But it is required to verify all the simple closed paths with length 4 for any pair of players. Then Hino (2011) gave an improved condition for detecting potential games, which has a lower complexity than that of Monderer and Shapley (1996) due to that only the adjacent pairs of strategies of two players need to check. In Ui (2000), it is proved that a game is potential if and only if the payoff functions coincide with the Shapley value of a particular class of cooperative games indexed by the set of strategy profiles. Game decomposition is an important method for potential games (Candogan, Menache, Ozdaglar, \& Parrilo, 2011; Hwang, \& Rey-Bellet 2011; Sandholm, 2010) and some new necessary and sufficient conditions for detecting potential games are obtained. Sandholm (2010) established connections between his results and that in Ui (2000). But the number of the obtained verification equations is not the minimum. In Sandholm (2010), it is proved that a finite game is a potential game if and only if, in each of the component games, all active players have identical payoff functions, and that in this case, the potential function can be constructed.

Recently, Cheng (2014) developed a novel method, based on the semi-tensor product of matrices, to deal with games including potential games, networked games and evolutionary games (Cheng, 2014; Cheng, Xu \& Qi, 2014; Cheng, Xu, He, Qi, 2014; Cheng, He, Qi, \& Xu, 2015; Guo, Wang, \& Li, 2013). In Cheng (2014), a lin- 
ear system, called potential equation, is proposed, and then it is proved that the game is potential if and only if the potential equation is solvable. With a solution of the potential equation, the potential function can be directly calculated.

A natural question is how to establish the connection between the potential equation and the other criteria of potential games. Moreover, an interesting problem is how to get the verification equations with the minimum number. In this paper, we further investigate the solvability of the potential equation. An equivalence transformation is constructed to convert the augmented matrix of the potential equation into the reduced row echelon form. Based on this technique, some new necessary and sufficient conditions for potential games are obtained. For potential games, a new formula to calculate the potential functions is proposed. Based on the obtained results, it is revealed the connection between the potential equation and the results in Hino (2011) and Sandholm (2010).

Throughout the paper, we denote the $k \times k$ identity matrix by $I_{k}$, the $i$-th column of $I_{k}$ by $\delta_{k}^{i}$, the $n$-dimensional column vector whose entries are all equal to 1 by $\mathbf{1}_{k}$, Kronecker product by $\otimes$ and the real number field by $\mathbb{R}$.

\section{Preliminaries}

Definition 1 (Monderer \& Shapley 1996) A finite game is a triple $\mathcal{G}=(\mathcal{N}, \mathcal{S}, \mathcal{C})$, where

(i) $\mathcal{N}=\{1,2, \cdots, n\}$ is the set of players;

(ii) $\mathcal{S}=\mathcal{S}_{1} \times \mathcal{S}_{2} \times \cdots \times \mathcal{S}_{n}$ is the strategy set, where each $\mathcal{S}_{i}=\left\{s_{1}^{i}, s_{2}^{i}, \cdots, s_{k_{i}}^{i}\right\}$ is the strategy set of player $i$;

(iii) $\mathcal{C}=\left\{c_{1}, c_{2}, \cdots, c_{n}\right\}$ is the set of payoff functions, where every $c_{i}: \mathcal{S} \rightarrow \mathbb{R}$ is the payoff function of player $i$.

Let $c_{i_{1} i_{2} \cdots i_{n}}^{\mu}=c_{\mu}\left(s_{i_{1}}^{1}, s_{i_{2}}^{2}, \cdots, s_{i_{n}}^{n}\right)$ where $1 \leq i_{s} \leq k_{s}$ and $s \stackrel{1}{=} 1,2, \cdots, n$. Then the finite game can be described by the arrays

$$
C_{\mu}=\left\{c_{i_{1} i_{2} \cdots i_{n}}^{\mu} \mid 1 \leq i_{s} \leq k_{s}, s=1,2, \cdots, n\right\}
$$

with $\mu=1,2, \cdots, n$. Particularly, for a 2-player game, the $k_{1} \times k_{2}$ matrices $C_{1}=\left(c_{i j}^{1}\right)$ and $C_{2}=\left(c_{i j}^{2}\right)$ are payoffs of players 1 and 2 respectively. Therefore, a 2 player finite game is also called a bi-matrix game, which is usually denoted by the simple notation $\mathcal{G}=\left(C_{1}, C_{2}\right)$.

Definition 2 (Monderer 8 Shapley 1996) A finite game $\mathcal{G}=(\mathcal{N}, \mathcal{S}, \mathcal{C})$ is said to be potential if there exists a function $p: \mathcal{S} \rightarrow \mathbb{R}$, called the potential function, such that $c_{i}\left(x, s^{-i}\right)-c_{i}\left(y, s^{-i}\right)=p\left(x, s^{-i}\right)-p\left(y,, s^{-i}\right)$ for all $x, y \in \mathcal{S}_{i}, s^{-i} \in \mathcal{S}^{-i}$ and $i=1,2, \cdots, n$, where $\mathcal{S}^{-i}=\mathcal{S}_{1} \times \cdots \times \mathcal{S}_{i-1} \times \mathcal{S}_{i+1} \times \cdots \times \mathcal{S}_{n}$.

Definition 3 (Cheng, Qi, \& Li, 2011). Assume $A \in$ $\mathbb{R}^{m \times n}, B \in \mathbb{R}^{p \times q}$. Let $\alpha=\operatorname{lcm}(n, p)$ be the least com- mon multiple of $n$ and $p$. The left semi-tensor product of $A$ and $B$ is defined as $A \ltimes B=\left(A \otimes I_{\frac{\alpha}{n}}\right)\left(B \otimes I_{\frac{\alpha}{p}}\right)$.

Since the left semi-tensor product is a generalization of the traditional matrix product, the left semi-tensor product $A \ltimes B$ can be directly written as $A B$. Identifying each strategy $s_{j}^{i}$ with the logical vector $\delta_{k_{i}}^{j}$ for $i=1,2, \cdots, n$ and $j=1,2, \cdots, k_{i}$, Cheng (2014) gave a new expression of the payoff functions using the left semi-tensor product.

Lemma 4 (Cheng, 2014) Let $x_{i} \in \mathcal{S}_{i}$ be any strategy expressed in the form of logical vectors. Then, for any payoff function $c_{i}$ of a finite game $\mathcal{G}$ shown in Definition 1 , there exists a unique row vector $V_{i}^{c} \in \mathbb{R}^{n}$ such that

$$
c_{i}\left(x_{1}, x_{2}, \cdots, x_{n}\right)=V_{i}^{c} x_{1} x_{2} \cdots x_{n},
$$

where $V_{i}^{c}$ is called the structure vector of $c_{i}$ and $i=$ $1,2, \cdots, n$.

Remark 5 It is easy to see that $V_{i}^{c}$ is just the row vector composed of the elements of $C_{i}$ in the lexicographic order (see $(1))$. Let $C=\left[\left(V_{1}^{c}\right)^{\mathrm{T}},\left(V_{2}^{c}\right)^{\mathrm{T}}, \cdots,\left(V_{n}^{c}\right)^{\mathrm{T}}\right]^{\mathrm{T}}$. Then $C$ is just the payoff matrix of $\mathcal{G}$ proposed by Cheng (2014).

Without loss of generality, we assume $k_{i}=k$ for all $i=1,2, \cdots, n$. In Cheng (2014), the potential equation is proposed as follows:

$$
\Psi \xi=b,
$$

where

$$
\Psi=\left[\begin{array}{ccccc}
-\Psi_{1} & \Psi_{2} & & & \\
-\Psi_{1} & \Psi_{3} & \\
\vdots & & \ddots & \\
-\Psi_{1} & & & \Psi_{n}
\end{array}\right], \xi=\left[\begin{array}{c}
\xi_{1} \\
\xi_{2} \\
\vdots \\
\xi_{n}
\end{array}\right], b=\left[\begin{array}{c}
\left(V_{2}^{c}-V_{1}^{c}\right)^{\mathrm{T}} \\
\left(V_{3}^{c}-V_{1}^{c}\right)^{\mathrm{T}} \\
\vdots \\
\left(V_{n}^{c}-V_{1}^{c}\right)^{\mathrm{T}}
\end{array}\right]
$$

and $\Psi_{i}=I_{k^{i-1}} \otimes \mathbf{1}_{k} \otimes I_{k^{n-i}}$ for each $i=1,2, \cdots, n$.

Lemma 6 (Cheng, 2014) A finite game $\mathcal{G}$ shown in Definition 1 is a potential game if and only if the potential equation (3) has a solution $\xi$. Moreover, as (3) holds, the potential function $p$ can be calculated by

$$
\left(V^{p}\right)^{\mathrm{T}}=\left(V_{1}^{c}\right)^{\mathrm{T}}-\left(\mathbf{1}_{k} \otimes I_{k^{n-1}}\right) \xi_{1} .
$$

\section{Bi-matrix games}

In this section, we consider the 2-player finite game $\mathcal{G}=$ $\left(C_{1}, C_{2}\right)$, where $C_{i} \in \mathbb{R}^{k_{1} \times k_{2}}$ for $i=1,2$. In this special case, the coefficients of the potential equation (3) become

$$
\Psi=\left[-\mathbf{1}_{k_{1}} \otimes I_{k_{2}} \quad I_{k_{1}} \otimes \mathbf{1}_{k_{2}}\right], \quad b=\left(V_{2}^{c}-V_{1}^{c}\right)^{\mathrm{T}} .
$$


Before the main results of this section, we first introduce a basic property on Kronecker product.

Lemma 7 (Horn, 1994) Let $\mathrm{V}_{\mathrm{r}}(X)$ denotes the vectorization of the matrix $X$ formed by stacking the rows of $X$ into a single column vector. Then

$$
\mathrm{V}_{\mathrm{r}}(A B C)=\left(A \otimes C^{\mathrm{T}}\right) \mathrm{V}_{\mathrm{r}}(B) .
$$

In particular, we have

$$
\mathrm{V}_{\mathrm{r}}(A B)=(A \otimes I) \mathrm{V}_{\mathrm{r}}(B) .
$$

Theorem 8 Set $B_{k}=\left[I_{k-1},-\mathbf{1}_{k-1}\right] \in \mathbb{R}^{(k-1) \times k}$. The bi-matrix game $\mathcal{G}=\left(C_{1}, C_{2}\right)$ is potential if and only if

$$
B_{k_{1}}\left(C_{2}-C_{1}\right) B_{k_{2}}^{\mathrm{T}}=0
$$

where $C_{1}, C_{2} \in \mathbb{R}^{k_{1} \times k_{2}}$. Moreover, as (9) holds, the matrix form of each potential function is

$$
P=C_{1}+\left[\begin{array}{ll}
0_{k_{1} \times\left(k_{1}-1\right)} & \mathbf{1}_{k_{1}}
\end{array}\right]\left(C_{2}-C_{1}\right)+\lambda \mathbf{1}_{k_{1}} \mathbf{1}_{k_{2}}^{\mathrm{T}},
$$

where $\lambda \in \mathbb{R}$ is an arbitrary number.

Proof. Let $D_{k}=\left[I_{k-1}, 0\right] \in \mathbb{R}^{(k-1) \times k}$. Then it is easy to check that

$$
B_{k} D_{k}^{\mathrm{T}}=I_{k-1}, \quad D_{k} \delta_{k}^{k}=B_{k} \mathbf{1}_{k}=0
$$

Let

$$
\begin{aligned}
& E=\left[-\delta_{k_{1}}^{k_{1}} \otimes I_{k_{2}}, B_{k_{1}}^{\mathrm{T}} \otimes \delta_{k_{2}}^{k_{2}}, B_{k_{1}}^{\mathrm{T}} \otimes B_{k_{2}}^{\mathrm{T}}\right]^{\mathrm{T}} \in \mathbb{R}^{k_{1} k_{2} \times k_{1} k_{2}}, \\
& F=\left[-\mathbf{1}_{k_{1}} \otimes I_{k_{2}}, \quad D_{k_{1}}^{\mathrm{T}} \otimes \mathbf{1}_{k_{2}}, D_{k_{1}}^{\mathrm{T}} \otimes D_{k_{2}}^{\mathrm{T}}\right] \in \mathbb{R}^{k_{1} k_{2} \times k_{1} k_{2}} .
\end{aligned}
$$

By (11), a straightforward computation shows that

$$
E F=I_{k_{1} k_{2}} .
$$

Eq. (14) shows that $E$ is nonsingular. So the potential equation is equivalent to

$$
E \Psi \xi=E b .
$$

With simple calculations, we have

$$
\begin{aligned}
& E\left[\begin{array}{ll}
\Psi & b
\end{array}\right]=\left[\begin{array}{c}
-\left(\delta_{k_{1}}^{k_{1}}\right)^{\mathrm{T}} \otimes I_{k_{2}} \\
B_{k_{1}} \otimes\left(\delta_{k_{2}}^{k_{2}}\right)^{\mathrm{T}} \\
B_{k_{1}} \otimes B_{k_{2}}
\end{array}\right]\left[\begin{array}{lll}
-\mathbf{1}_{k_{1}} \otimes I_{k_{2}} & I_{k_{1}} \otimes \mathbf{1}_{k_{2}} & b
\end{array}\right]
\end{aligned}
$$

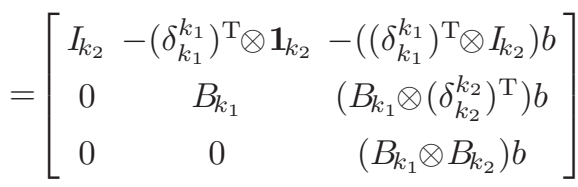

$$
=\left[\begin{array}{cccc}
I_{k_{2}} & 0 & -\mathbf{1}_{k_{2}} & -\left(\left(\delta_{k_{1}}^{k_{1}}\right)^{\mathrm{T}} \otimes I_{k_{2}}\right) b \\
0 & I_{k_{1}-1} & -\mathbf{1}_{k_{1}-1} & \left(B_{k_{1}} \otimes\left(\delta_{k_{2}}^{k_{2}}\right)^{\mathrm{T}}\right) b \\
0 & 0 & 0 & \left(B_{k_{1}} \otimes B_{k_{2}}\right) b
\end{array}\right] .
$$

From (16), it follows that (15) is solvable if and only if

$$
\left(B_{k_{1}} \otimes B_{k_{2}}\right) b=0 \text {, }
$$

whose matrix form is just (9) by (7). As (9) holds, from (16), we get

$$
\xi_{1}=-\left(\left(\delta_{k_{1}}^{k_{1}}\right)^{\mathrm{T}} \otimes I_{k_{2}}\right)\left(V_{2}^{c}-V_{1}^{c}\right)^{\mathrm{T}}-c \mathbf{1}_{k_{2}},
$$

where $c$ is an arbitrary constant. Substituting (18) into (5) yields

$$
\left(V^{p}\right)^{\mathrm{T}}=\left(V_{1}^{c}\right)^{\mathrm{T}}+\left(\mathbf{1}_{k_{1}}\left(\delta_{k_{1}}^{k_{1}}\right)^{\mathrm{T}} \otimes I_{k_{2}}\right)\left(V_{2}^{c}-V_{1}^{c}\right)^{\mathrm{T}}+c \mathbf{1}_{k_{1} k_{2}} .
$$

Using (8), from (19), we get

$$
P=C_{1}+\mathbf{1}_{k_{1}}\left(\delta_{k_{1}}^{k_{1}}\right)^{\mathrm{T}}\left(C_{2}-C_{1}\right)+c \mathbf{1}_{k_{1}} \mathbf{1}_{k_{2}}^{\mathrm{T}},
$$

which is just (10).

Corollary 9 Given a bi-matrix game $\mathcal{G}=\left(C_{1}, C_{2}\right)$, we write the relative payoffs in the matrix form as

$$
R=\left(r_{i j}\right)=C_{2}-C_{1}=\left[\begin{array}{cc}
R_{1} & \eta \\
\zeta^{\mathrm{T}} & r_{k_{1} k_{2}}
\end{array}\right],
$$

where $R_{1} \in \mathbb{R}^{\left(k_{1}-1\right) \times\left(k_{2}-1\right)}$. Then $\mathcal{G}$ is potential if and only if

$$
R_{1}-\mathbf{1}_{k_{1}-1} \zeta^{\mathrm{T}}-\eta \mathbf{1}_{k_{2}-1}^{\mathrm{T}}+r_{k_{1} k_{2}} \mathbf{1}_{k_{1}-1} \mathbf{1}_{k_{2}-1}^{\mathrm{T}}=0,
$$

namely,

$r_{i j}-r_{i k_{2}}-r_{k_{1} j}+r_{k_{1} k_{2}}=0$

for all $i=1,2, \cdots, k_{1}-1$ and $j=1,2, \cdots, k_{2}-1$.

Proof. Considering

$$
\begin{aligned}
B_{k_{1}} R B_{k_{2}}^{\mathrm{T}} & =\left[I_{k_{1}-1},-\mathbf{1}_{k_{1}-1}\right]\left[\begin{array}{cc}
R_{1} & \eta \\
\zeta^{\mathrm{T}} & r_{k_{1} k_{2}}
\end{array}\right]\left[\begin{array}{c}
I_{k_{1}-1} \\
-\mathbf{1}_{k_{1}-1}^{\mathrm{T}}
\end{array}\right] \\
& =R_{1}-\mathbf{1}_{k_{1}-1} \zeta^{\mathrm{T}}-\eta \mathbf{1}_{k_{2}-1}^{\mathrm{T}}+r_{k_{1} k_{2}} \mathbf{1}_{k_{1}-1} \mathbf{1}_{k_{2}-1}^{\mathrm{T}}
\end{aligned}
$$

we get the corollary from Theorem 8 .

The condition (23) of Corollary 9 is similar to the condition proposed in Theorem 3 of Hino (2011). It should be noted that Theorem 3 of Hino (2011) considers finite weighted potential games. Here, for the convenience of comparing our result with that in Hino (2011), we rewrite Theorem 3 of Hino (2011) for the special case of 2-player potential games in the language of relative payoff matrix as follows: 
Proposition 10 (see Theorem 3 of Hino, 2011) The bimatrix game $\mathcal{G}=\left(C_{1}, C_{2}\right)$ is potential if and only if

$$
r_{i j}-r_{i+1, j}-r_{i, j+1}+r_{i+1, j+1}=0
$$

for all $i=1,2, \cdots, k_{1}-1$ and $j=1,2, \cdots, k_{2}-1$.

The original four-cycle condition proposed by Monderer \& Shapley (1996) is rewritten as:

Proposition 11 (see Corollary 2.9 of Monderer \& Shapley, 1996) The bi-matrix game $\mathcal{G}=\left(C_{1}, C_{2}\right)$ is potential if and only if

$$
r_{i j}-r_{i^{\prime}, j}-r_{i, j^{\prime}}+r_{i^{\prime}, j^{\prime}}=0
$$

for all $i, i^{\prime}=1,2, \cdots, k_{1}$ and $j, j^{\prime}=1,2, \cdots, k_{2}$.

Obviously, our condition (23) is different from Hino's condition (25) and they have the same complexity, but (26) has a larger complexity (Hino, 2011).

Remark 12 From (9) or (17), we see that, given the strategy set for bi-matrix games, the set of all the relative payoff matrices of potential bi-matrix games is a $\left(k_{1}+\right.$ $k_{2}-1$ )-dimensional subspace, which is isomorphic to

$$
\mathcal{P}=\left\{b \in \mathbb{R}^{k_{1} k_{2}} \mid\left(B_{k_{1}} \otimes B_{k_{2}}\right) b=0\right\} .
$$

Here, we call $\mathcal{P}$ the potential subspace. If a bi-matrix game $\mathcal{G}=\left(C_{1}, C_{2}\right)$ is not a potential game, then one can use the orthogonal projection onto $\mathcal{P}$ to yield corresponding potential games.

The basic result on orthogonal projection is stated as follows:

Lemma 13 (see page 430 of Meyer, 2000) Consider a linear subspace of $\mathbb{R}^{n}$ as follows:

$$
\mathcal{X}=\left\{v \in \mathbb{R}^{n} \mid B v=0\right\} .
$$

If $B$ has a full row rank, then the orthogonal projection of $u$ onto $\mathcal{X}$ is

$$
\operatorname{Proj}_{\mathcal{X}} u=\left(I_{n}-B^{\mathrm{T}}\left(B B^{\mathrm{T}}\right)^{-1} B\right) u .
$$

Now we consider the orthogonal projection onto the potential subspace.

Lemma 14 Consider a bi-matrix game $\mathcal{G}=\left(C_{1}, C_{2}\right)$, where $C_{1}, C_{2} \in \mathbb{R}^{k_{1} \times k_{2}}$. Denote the relative payoff matrix by $R=\left(r_{i j}\right)=C_{2}-C_{1}$ and let $H_{k}=I_{k}-\frac{1}{k} \mathbf{1}_{k} \mathbf{1}_{k}^{\mathrm{T}}$. Then

$$
\operatorname{Proj}_{\mathcal{P}} \mathrm{V}_{\mathrm{r}}(R)=\left(I_{k_{1} k_{2}}-H_{k_{1}} \otimes H_{k_{2}}\right) \mathrm{V}_{\mathrm{r}}(R) .
$$

Proof. Let $\tilde{B}=B_{k_{1}} \otimes B_{k_{2}}$. By Lemma 13, we have

$$
\begin{aligned}
& \operatorname{Proj}_{\mathcal{P}} \mathrm{V}_{\mathrm{r}}(R) \\
= & \left(I_{k_{1} k_{2}}-\tilde{B}^{\mathrm{T}}\left(\tilde{B} \tilde{B}^{\mathrm{T}}\right)^{-1} \tilde{B}\right) \mathrm{V}_{\mathrm{r}}(R) \\
= & \left(I_{k_{1} k_{2}}-\left(B_{k_{1}}^{\mathrm{T}}\left(B_{k_{1}} B_{k_{1}}^{\mathrm{T}}\right)^{-1} B_{k_{1}}\right) \otimes\left(B_{k_{2}}^{\mathrm{T}}\left(B_{k} B_{k_{2}}^{\mathrm{T}}\right)^{-1} B_{k_{2}}\right)\right) \mathrm{V}_{\mathrm{r}}(R) .
\end{aligned}
$$

A straightforward computation shows that

$$
\begin{aligned}
& B_{k}^{\mathrm{T}}\left(B_{k} B_{k}^{\mathrm{T}}\right)^{-1} B_{k} \\
& =\left[\begin{array}{c}
I_{k-1} \\
-\mathbf{1}_{k-1}^{\mathrm{T}}
\end{array}\right]\left(I_{k-1}+\mathbf{1}_{k-1} \mathbf{1}_{k-1}^{\mathrm{T}}\right)^{-1}\left[\begin{array}{ll}
I_{k-1} & -\mathbf{1}_{k-1}
\end{array}\right] \\
& =\left[\begin{array}{c}
I_{k-1} \\
-\mathbf{1}_{k-1}^{\mathrm{T}}
\end{array}\right]\left(I_{k-1}-\frac{1}{k} \mathbf{1}_{k-1} \mathbf{1}_{k-1}^{\mathrm{T}}\right)\left[\begin{array}{ll}
I_{k-1} & -\mathbf{1}_{k-1}
\end{array}\right] \\
& =\left[\begin{array}{cc}
I_{k-1}-\frac{1}{k} \mathbf{1}_{k-1} \mathbf{1}_{k-1}^{\mathrm{T}} & -\frac{1}{k} \mathbf{1}_{k-1} \\
-\frac{1}{k} \mathbf{1}_{k-1}^{\mathrm{T}} & \frac{k-1}{k}
\end{array}\right] \\
& =I_{k}-\frac{1}{k} \mathbf{1}_{k} \mathbf{1}_{k}^{\mathrm{T}}=H_{k} \text {. }
\end{aligned}
$$

From (31) and (32), it follows that (30) holds.

Theorem 15 Consider a bi-matrix game $\mathcal{G}=\left(C_{1}, C_{2}\right)$, where $C_{1}, C_{2} \in \mathbb{R}^{k_{1} \times k_{2}}$. Let the relative payoff matrix be $R=\left(r_{i j}\right)=C_{2}-C_{1}$. Then the following statements are equivalent:

(i) $\mathcal{G}$ is a potential game;

(ii) $H_{k_{1}} R H_{k_{2}}=0$, where $H_{k}=I_{k}-\frac{1}{k} \mathbf{1}_{k} \mathbf{1}_{k}^{\mathrm{T}}$;

(iii) $r_{i j}=r_{i-\text { ave }}+r^{j-\text { ave }}-r_{\text {ave }}$ for all $i=1,2, \cdots, k_{1}$ and $j=1,2, \cdots, k_{2}$, where

$$
\begin{gathered}
r_{i-\text { ave }}=\frac{1}{k_{2}} \sum_{\mu=1}^{k_{2}} r_{i \mu}, \quad r^{j-\text { ave }}=\frac{1}{k_{1}} \sum_{\lambda=1}^{k_{1}} r_{\lambda j}, \\
r_{\text {ave }}=\frac{1}{k_{1} k_{2}} \sum_{\lambda=1}^{k_{1}} \sum_{\mu=1}^{k_{2}} r_{\lambda \mu} .
\end{gathered}
$$

Proof. Obviously, $\mathcal{G}$ is a potential game if and only if $\operatorname{Proj}_{\mathcal{P}} \mathrm{V}_{\mathrm{r}}(R)=\mathrm{V}_{\mathrm{r}}(R)$, where $\mathcal{P}$ is the potential subspace. Further by Lemma 14, we have that $\mathcal{G}$ is potential if and only if $\left(H_{k_{1}} \otimes H_{k_{2}}\right) \mathrm{V}_{\mathrm{r}}(R)=0$, i.e. $H_{k} R H_{k}=0$. Moreover, a straightforward calculation shows that

$$
\begin{aligned}
& H_{k} R H_{k} \\
= & \left(I_{k_{1}}-\frac{1}{k_{1}} \mathbf{1}_{k_{1}} \mathbf{1}_{k_{1}}^{\mathrm{T}}\right) R\left(I_{k_{2}}-\frac{1}{k_{2}} \mathbf{1}_{k_{2}} \mathbf{1}_{k_{2}}^{\mathrm{T}}\right) \\
= & R-\frac{1}{k_{1}} \mathbf{1}_{k_{1}} \mathbf{1}_{k_{1}}^{\mathrm{T}} R-\frac{1}{k_{2}} R \mathbf{1}_{k_{2}} \mathbf{1}_{k_{2}}^{\mathrm{T}}+\frac{\mathbf{1}_{k_{1}}^{\mathrm{T}} R \mathbf{1}_{k_{2}}}{k_{1} k_{2}} \mathbf{1}_{k_{1}} \mathbf{1}_{k_{2}}^{\mathrm{T}} .
\end{aligned}
$$

From (33)-(35), the equivalence between (ii) and (iii) follows. 
Remark 16 For the case of $k_{1}=k_{2}$, Sandholm (2010) obtained the results of Theorem 15 using the method of game decomposition. A similar result can be seen in Proposition 2.14 of Hwang \& Rey-Bellet (2011). But here, we get the results from the potential equation. Therefore, we have established a connection between the potential equation and the results obtained by Sandholm (2010). From (33) and (34), we see that $r_{i-a v e}$ is the average relative payoff for given strategy $s_{i}^{1} \in \mathcal{S}_{1}$, $r^{j-a v e}$ is the average relative payoff for given strategy $s_{j}^{2} \in \mathcal{S}_{2}$ and $r^{\text {ave }}$ is the average relative payoff of all the strategies. Therefore, Theorem 15 displays an economic meaning of potential games.

\section{The General Potential Equation}

In this section, we consider the general potential equation for multi-player games and give new detecting conditions for potential games.

Multiplying (3) on the left by

$$
\left[\begin{array}{cc}
0 & 1 \\
-I_{n-1} & \mathbf{1}_{n-1}
\end{array}\right] \otimes I_{k^{n}},
$$

we get the equivalent equation

$$
\left[\begin{array}{ccccc}
-\Psi_{1} & & & & \Psi_{n} \\
& -\Psi_{2} & & & \Psi_{n} \\
& & \ddots & & \vdots \\
& & & -\Psi_{n-1} & \Psi_{n}
\end{array}\right]\left[\begin{array}{c}
\xi_{1} \\
\xi_{2} \\
\vdots \\
\xi_{n}
\end{array}\right]=\left[\begin{array}{c}
\left(V_{n}^{c}-V_{1}^{c}\right)^{\mathrm{T}} \\
\left(V_{n}^{c}-V_{2}^{c}\right)^{\mathrm{T}} \\
\vdots \\
\left(V_{n}^{c}-V_{n-1}^{c}\right)^{\mathrm{T}}
\end{array}\right]
$$

Construct nonsingular matrix $T=\left[\begin{array}{lll}T_{1}^{\mathrm{T}} & T_{2}^{\mathrm{T}} & T_{3}^{\mathrm{T}}\end{array}\right]^{\mathrm{T}}$, where

$$
T_{i}=\left[\begin{array}{llll}
-T_{i 1} & & & \\
& -T_{i 2} & & \\
& & \ddots & \\
& & & -T_{i, n-1}
\end{array}\right]
$$

with

$$
\begin{aligned}
& T_{1 j}=I_{k^{j-1}} \otimes\left(\delta_{k}^{k}\right)^{\mathrm{T}} \otimes I_{k^{n-j}}, \\
& T_{2 j}=I_{k^{j-1}} \otimes B_{k} \otimes I_{k^{n-j-1}} \otimes\left(\delta_{k}^{k}\right)^{\mathrm{T}}, \\
& T_{3 j}=I_{k^{j-1}} \otimes B_{k} \otimes I_{k^{n-j-1}} \otimes B_{k}
\end{aligned}
$$

for all $j=1,2, \cdots, n$. It is easy to check that

$$
\begin{aligned}
T_{1 j} \Psi_{j} & =\left(I_{k^{j-1}} \otimes\left(\delta_{k}^{k}\right)^{\mathrm{T}} \otimes I_{k^{n-j}}\right)\left(I_{k^{j-1}} \otimes \mathbf{1}_{k} \otimes I_{k^{n-j}}\right)=I_{k^{n-1}} \\
T_{1 j} \Psi_{n} & =\left(I_{k^{j-1}} \otimes\left(\delta_{k}^{k}\right)^{\mathrm{T}} \otimes I_{k^{n-j}}\right)\left(I_{k^{n-1}} \otimes \mathbf{1}_{k}\right) \\
& =I_{k^{j-1}} \otimes\left(\delta_{k}^{k}\right)^{\mathrm{T}} \otimes I_{k^{n-j-1}} \otimes \mathbf{1}_{k} \\
T_{2 j} \Psi_{j} & =\left(I_{k^{j-1}} \otimes B_{k} \otimes I_{k^{n-j-1}} \otimes\left(\delta_{k}^{k}\right)^{\mathrm{T}}\right)\left(I_{k^{j-1}} \otimes \mathbf{1}_{k} \otimes I_{k^{n-j}}\right)=0 \\
T_{2 j} \Psi_{n} & =\left(I_{k^{j-1}} \otimes B_{k} \otimes I_{k^{n-j-1}} \otimes\left(\delta_{k}^{k}\right)^{\mathrm{T}}\right)\left(I_{k^{n-1}} \otimes \mathbf{1}_{k}\right) \\
& =I_{k^{j-1}} \otimes B_{k} \otimes I_{k^{n-j-1}} \\
T_{3 j} \Psi_{j} & =\left(I_{k^{j-1}} \otimes B_{k} \otimes I_{k^{n-j-1}} \otimes B_{k}\right)\left(I_{k^{j-1}} \otimes \mathbf{1}_{k} \otimes I_{k^{n-j}}\right)=0 \\
T_{3 j} \Psi_{n} & =\left(I_{k^{j-1}} \otimes B_{k} \otimes I_{k^{n-j-1}} \otimes B_{k}\right)\left(I_{k^{n-1}} \otimes \mathbf{1}_{k}\right)=0
\end{aligned}
$$

So, multiplying (37) on the left by $T$ yields

$$
\left[\begin{array}{cccc}
I_{k^{n-1}} & & -\left(\delta_{k}^{k}\right)^{\mathrm{T}} \otimes I_{k^{n-2}} \otimes \mathbf{1}_{k} \\
& \ddots & \vdots \\
& & I_{k^{n-1}} & -I_{k^{n-2}} \otimes\left(\delta_{k}^{k}\right)^{\mathrm{T}} \otimes \mathbf{1}_{k} \\
0 & \cdots & 0 & -B_{k} \otimes I_{k^{n-2}} \\
0 & \cdots & 0 & -I_{k} \otimes B_{k} \otimes I_{k^{n-3}} \\
\vdots & \ddots & \vdots & \vdots \\
0 & \cdots & 0 & -I_{k^{n-2}} \otimes B_{k} \\
0 & \cdots & 0 & 0
\end{array}\right]\left[\begin{array}{c}
\xi_{1} \\
\xi_{2} \\
\vdots \\
\xi_{n}
\end{array}\right]=T\left[\begin{array}{c}
\left(V_{n}^{c}-V_{1}^{c}\right)^{\mathrm{T}} \\
\left(V_{n}^{c}-V_{2}^{c}\right)^{\mathrm{T}} \\
\vdots \\
\left(V_{n}^{c}-V_{n-1}^{c}\right)^{\mathrm{T}}
\end{array}\right],
$$

that is,

$$
\left[\begin{array}{cc}
I_{(n-1) k^{n-1}} & \Gamma \\
0 & \Phi \\
0 & 0
\end{array}\right]\left[\begin{array}{c}
\tilde{\xi} \\
\xi_{n}
\end{array}\right]=\left[\begin{array}{c}
T_{1} \tilde{b} \\
T_{2} \tilde{b} \\
T_{3} \tilde{b}
\end{array}\right],
$$

where $\tilde{\xi}=\left[\xi_{1}^{\mathrm{T}}, \cdots, \xi_{n-1}^{\mathrm{T}}\right]^{\mathrm{T}}, \tilde{b}=\left[V_{n}^{c}-V_{1}^{c}, \cdots, V_{n}^{c}-V_{n-1}^{c}\right]^{\mathrm{T}}$, $\Phi=\left[\Phi_{1}^{\mathrm{T}}, \Phi_{2}^{\mathrm{T}}, \cdots, \Phi_{n-1}^{\mathrm{T}}\right]^{\mathrm{T}}, \Gamma=\left[\Gamma_{1}^{\mathrm{T}}, \Gamma_{2}^{\mathrm{T}}, \cdots, \Gamma_{n-1}^{\mathrm{T}}\right]^{\mathrm{T}}$ with

$$
\Phi_{i}=-I_{k^{i-1}} \otimes B_{k} \otimes I_{k^{n-i-1}}, \quad \Gamma_{i}=-I_{k^{i-1}} \otimes\left(\delta_{k}^{k}\right)^{\mathrm{T}} \otimes I_{k^{n-1-i}} \otimes \mathbf{1}_{k}
$$

for each $i=1,2, \cdots, n-1$.

By (49), we get the proposition as follows:

Proposition 17 The finite game $G$ is potential if and only if $T_{3} \tilde{b}=0$ and the linear equation

$$
\Phi \xi_{n}=T_{2} \tilde{b}
$$

has a solution $\xi_{n}$. 
In the following, we consider (51). Let

$$
S=\left[\begin{array}{l}
S_{1} \\
S_{2}
\end{array}\right]=\left[\begin{array}{cccccc}
N_{11} & N_{12} & N_{13} & \cdots & N_{1, n-2} & N_{1, n-1} \\
\hline M_{21} & L_{22} & L_{23} & \cdots & L_{2, n-2} & L_{2, n-1} \\
& M_{32} & L_{33} & \cdots & L_{3, n-2} & L_{3, n-1} \\
& & \ddots & \ddots & \vdots & \vdots \\
& & & \ddots & L_{n-2, n-2} & L_{n-2, n-1} \\
& & & & M_{n-1, n-2} & L_{n-1, n-1}
\end{array}\right],
$$

where

$$
\begin{aligned}
& N_{i j}=D_{k^{n-i}}\left(I_{k^{j-i}} \otimes D_{k}^{\mathrm{T}} \otimes \mathbf{1}_{k^{n-j-1}}\left(\delta_{k^{n-j-1}}^{k^{n-j-1}}\right)^{\mathrm{T}}\right), \\
& L_{i j}=-I_{k^{i-2}} \otimes B_{k} \otimes N_{i j}, \\
& M_{i, i-1}=I_{k^{i-2}(k-1)} \otimes B_{k^{n-i}}
\end{aligned}
$$

for all $i=1,2, \cdots, n-1$ and $j=i, i+1, \cdots, n-1$.

It is easy to check that $S$ is a square matrix with order $(n-1)(k-1) k^{n-2}$. In order to prove $S$ is nonsingular, we construct a square matrix as follows:

$$
U=\left[\begin{array}{ccccc}
-\Phi_{1} D_{k^{n-1}}^{\mathrm{T}} & G_{1} & & & \\
-\Phi_{2} D_{k^{n-1}}^{\mathrm{T}} & & G_{2} & & \\
\vdots & & & \ddots & \\
& & & & \\
-\Phi_{n-2} D_{k^{n-1}}^{\mathrm{T}} & & & G_{n-2} \\
-\Phi_{n-1} D_{k^{n-1}}^{\mathrm{T}} & & & & 0
\end{array}\right]
$$

where $G_{i}=I_{k^{i-1}(k-1)} \otimes D_{k^{n-i-1}}^{\mathrm{T}}$ for $i=1,2, \cdots, n-2$.

Lemma 18 For matrices $S$ and $U$, we have

$$
\begin{aligned}
& -\sum_{j=1}^{n-1} N_{1 j} \Phi_{j}=B_{k^{n-1}} \\
& M_{i, i-1} \Phi_{i-1}+\sum_{j=i}^{n-1} L_{i j} \Phi_{j}=0 \quad(i=2,3, \cdots, n-1) \\
& S U=I_{(n-1)(k-1) k^{n-2}} .
\end{aligned}
$$

Proof. See Appendix.

From (59), it follows that $S$ is nonsingular. Multiplying (51) on the left by $S$, we get an equivalent equation

$$
S \Phi \xi_{n}=S T_{2} \tilde{b} .
$$

Let $S \Phi=\left[\Upsilon_{1}^{\mathrm{T}}, \Upsilon_{2}^{\mathrm{T}}, \cdots, \Upsilon_{n-1}^{\mathrm{T}}\right]^{\mathrm{T}}$. From (57) and (58), it follows that $\Upsilon_{1}=B_{k^{n-1}}=\left[\begin{array}{lll}I_{k^{n-1}-1} & -\mathbf{1}_{k^{n-1}-1}\end{array}\right]$ and $\Upsilon_{i}=0$ for all $i=2,3, \cdots, n-1$. Thus the linear equation (60) is just

$$
\begin{aligned}
& {\left[\begin{array}{ll}
I_{k^{n-1}-1} & -\mathbf{1}_{k^{n-1}-1}
\end{array}\right] \xi_{n}=S_{1} T_{2} \tilde{b},} \\
& 0=S_{2} T_{2} \tilde{b} .
\end{aligned}
$$

Then we get the following proposition.

Proposition 19 The linear equation (51) has a solution $\xi_{n}$ if and only if $S_{2} T_{2} \tilde{b}=0$.

Theorem 20 Consider the finite game $\mathcal{G}=(\mathcal{N}, \mathcal{S}, \mathcal{C})$ described by Definition 1 with payoff functions in (2). $\mathcal{G}$ is potential if and only if

$$
\left[\begin{array}{c}
S_{2} T_{2} \\
T_{3}
\end{array}\right] \tilde{b}=0,
$$

where $\tilde{b}=\left[V_{n}^{c}-V_{1}^{c}, \cdots, V_{n}^{c}-V_{n-1}^{c}\right]^{\mathrm{T}}$ and the matrices $T_{2}, T_{3}$ and $S_{2}$ are shown in (38) and (52). Moreover, as (63) holds, a potential function is described by

$$
p\left(x_{1}, \cdots, x_{n}\right)=V^{p} x_{1} x_{2} \cdots x_{n},
$$

where

$$
\begin{gathered}
\left(V^{p}\right)^{\mathrm{T}}=\left(V_{1}^{c}\right)^{\mathrm{T}}+\left(\mathbf{1}_{k}\left(\delta_{k}^{k}\right)^{\mathrm{T}} \otimes I_{k^{n-1}}\right)\left(V_{n}^{c}-V_{1}^{c}\right)^{\mathrm{T}} \\
-\sum_{j=2}^{n-1}\left(\mathbf{1}_{k}\left(\delta_{k}^{k}\right)^{\mathrm{T}} \otimes I_{k^{j-2}} \otimes D_{k}^{\mathrm{T}} B_{k} \otimes \mathbf{1}_{k^{n-j}}\left(\delta_{k^{n-j}}^{k^{n-j}}\right)^{\mathrm{T}}\right) \\
\quad \cdot\left(V_{n}^{c}-V_{j}^{g}\right)^{\mathrm{T}}+c \mathbf{1}_{k^{n}} .
\end{gathered}
$$

Proof. From Proposition 17 and Proposition 19, it follows that $\mathcal{G}$ is potential if and only if (63) holds. Now, we compute the potential function. From (61), we get the

$$
\xi_{n}=\left[\begin{array}{c}
S_{1} \\
0
\end{array}\right] T_{2} \tilde{b}+c \mathbf{1}_{k^{n-1}},
$$

where $c$ is an arbitrary constant. From (52) and (53), it follows that

$S_{1}=D_{k^{n-1}} \tilde{S}_{1}$

where $\tilde{S}_{1}=\left[\tilde{S}_{11}, \tilde{S}_{12}, \cdots, \tilde{S}_{1, n-1}\right]$ with

$$
\tilde{S}_{1 j}=I_{k^{j-1}} \otimes D_{k}^{\mathrm{T}} \otimes \mathbf{1}_{k^{n-j-1}}\left(\delta_{k^{n-j-1}}^{k^{n-j-1}}\right)^{\mathrm{T}} .
$$

Considering the last row of $\tilde{S}_{1}$ is 0 , by (66) and (67), we have

$$
\xi_{n}=\tilde{S}_{1} T_{2} \tilde{b}+c \mathbf{1}_{k^{n-1}} .
$$

By the first equation of (48), we obtain that

$$
\begin{aligned}
\xi_{1} & =-T_{11}\left(V_{n}^{c}-V_{1}^{c}\right)^{\mathrm{T}}+\left(\left(\delta_{k}^{k}\right)^{\mathrm{T}} \otimes I_{k^{n-2}} \otimes \mathbf{1}_{k}\right) \xi_{n} \\
& =-T_{11}\left(V_{n}^{c}-V_{1}^{c}\right)^{\mathrm{T}}+\left(\left(\delta_{k}^{k}\right)^{\mathrm{T}} \otimes I_{k^{n-2}} \otimes \mathbf{1}_{k}\right)\left(\tilde{S} T_{1} T_{2} \tilde{b}+c \mathbf{1}_{k^{n-1}}\right) .(
\end{aligned}
$$


Substituting (70) into (5), we have

$$
\begin{aligned}
\left(V^{p}\right)^{\mathrm{T}}= & \left(V_{1}^{c}\right)^{\mathrm{T}}-\left(\mathbf{1}_{k} \otimes I_{k^{n-1}}\right) \xi_{1} \\
= & \left(V_{1}^{c}\right)^{\mathrm{T}}+\left(\mathbf{1}_{k} \otimes I_{k^{n-1}}\right) T_{11}\left(V_{n}^{c}-V_{1}^{c}\right)^{\mathrm{T}} \\
& -\left(\left(\delta_{k}^{k}\right)^{\mathrm{T}} \otimes I_{k^{n-2}} \otimes \mathbf{1}_{k}\right) \sum_{j=1}^{n-1} \tilde{S}_{1 j} T_{2 j}\left(V_{n}^{c}-V_{j}^{c}\right)^{\mathrm{T}}+c \mathbf{1}_{k^{n}} .
\end{aligned}
$$

Since $D_{k} \delta_{k}^{k}=0$, we have

$$
\begin{aligned}
& \left(\left(\delta_{k}^{k}\right)^{\mathrm{T}} \otimes I_{k^{n-2}} \otimes \mathbf{1}_{k}\right) \tilde{S}_{11} \\
= & \left(\left(\delta_{k}^{k}\right)^{\mathrm{T}} \otimes I_{k^{n-2}} \otimes \mathbf{1}_{k}\right)\left(D_{k}^{\mathrm{T}} \otimes \mathbf{1}_{k^{n-2}}\left(\delta_{k^{n-2}}^{k^{n-2}}\right)^{\mathrm{T}}\right)=0 .
\end{aligned}
$$

Substituting $T_{11}, T_{2 j}, \tilde{S}_{1 j}$ and (72) into (71) yields (65).

Remark 21 In Section 4 of Sandholm (2010), it is revealed that the minimal number of linear equations to test potential games is $(n-1) k^{n}-n k^{n-1}+1$ for a $n$ player games with $k$ strategies. However, for both the methods of Sandholm (2010) and Hino (2011), the number of equalities to be verified is $\frac{1}{2} n(n-1) k^{n-2}(k-1)^{2}$ (see the footnote of page 455 of Sandholm (2010)), which is much greater than the minimal number. Fortunately, using the potential equation in Cheng (2014), we get the minimal number of equations described by (63).

Theorem 22 For the finite game $\mathcal{G}=(\mathcal{N}, \mathcal{S}, \mathcal{C})$ described by Definition 1 with payoff functions shown in (2), the following statements are equivalent:

(i) $\mathcal{G}$ is potential;

(ii) equalities

$$
\begin{aligned}
& \left(I_{k^{i-1}} \otimes B_{k} \otimes I_{k^{n-i-1}} \otimes B_{k}\right)\left(V_{n}^{c}-V_{i}^{c}\right)^{\mathrm{T}}=0, \\
& \left(I_{k^{i-1}} \otimes B_{k} \otimes I_{k^{j-i-1}} \otimes B_{k} \otimes I_{k^{n-j-1}} \otimes\left(\delta_{k}^{k}\right)^{\mathrm{T}}\right)\left(V_{j}^{c}-V_{i}^{c}\right)^{\mathrm{T}}=0
\end{aligned}
$$

hold for all $1 \leq i<j \leq n-1$;

(iii) equalities

$$
\left(I_{k^{i-1}} \otimes B_{k} \otimes I_{k^{j-i-1}} \otimes B_{k} \otimes I_{k^{n-j}}\right)\left(V_{j}^{c}-V_{i}^{c}\right)^{\mathrm{T}}=0
$$

hold for all $1 \leq i<j \leq n$.

Proof. (i) $\Rightarrow$ (ii) Assume that $\mathcal{G}$ is potential. Then, from Proposition 17, it follows that $T_{3} \tilde{b}=0$ and the linear equation (51) is solvable. It is easy to check that $T_{3} \tilde{b}=0$ is just (73). Moreover, (51) implies that

$\left(I_{k^{s-1}} \otimes B_{k} \otimes I_{k^{n-s-1}}\right) \xi_{n}=\left(I_{k^{s-1}} \otimes B_{k} \otimes I_{k^{n-s-1}} \otimes\left(\delta_{k}^{k}\right)^{\mathrm{T}}\right)\left(V_{n}^{c}-V_{s}^{c}\right)^{\mathrm{T}}$.

Letting $s=i$ in (76) and multiplying (76) on the left by $I_{k^{j-1}} \otimes B_{k} \otimes I_{k^{n-j-1}}$ yield

$$
\begin{aligned}
& \left(I_{k^{i-1}} \otimes B_{k} \otimes I_{k^{j-i+1}} \otimes B_{k} \otimes I_{k^{n-j-1}}\right) \xi_{n} \\
= & \left(I_{k^{i-1}} \otimes B_{k} \otimes I_{k^{j-i+1}} \otimes B_{k} \otimes I_{k^{n-j-1}} \otimes\left(\delta_{k}^{k}\right)^{\mathrm{T}}\right)\left(V_{n}^{c}-V_{i}^{c}\right)^{\mathrm{T}} .
\end{aligned}
$$

Similarly, letting $s=j$ in (76) and multiplying (76) on the left by $I_{k^{i-1}} \otimes B_{k} \otimes I_{k^{n-i-1}}$ yield

$$
\begin{aligned}
& \left(I_{k^{i-1}} \otimes B_{k} \otimes I_{k^{j-i+1}} \otimes B_{k} \otimes I_{k^{n-j-1}}\right) \xi_{n} \\
= & \left(I_{k^{i-1}} \otimes B_{k} \otimes I_{k^{j-i+1}} \otimes B_{k} \otimes I_{k^{n-j-1}} \otimes\left(\delta_{k}^{k}\right)^{\mathrm{T}}\right)\left(V_{n}^{c}-V_{j}^{c}\right)^{\mathrm{T}} .
\end{aligned}
$$

Subtracting (78) from (77), we get (74).

(ii) $\Rightarrow$ (iii) As $j=n,(75)$ is just (73). For the case of $1 \leq i<j \leq n-1$, from (73) and (74), it follows that

$$
\begin{aligned}
& \left(I_{k^{i-1}} \otimes B_{k} \otimes I_{k^{j-i-1}} \otimes B_{k} \otimes I_{k^{n-j}}\right)\left(V_{j}^{c}-V_{i}^{c}\right)^{\mathrm{T}} \\
= & \left(I_{k^{i-1}} \otimes B_{k} \otimes I_{k^{j-i-1}} \otimes B_{k} \otimes I_{k^{n-j-1}} \otimes\left(D_{k}^{\mathrm{T}} B_{k}+\mathbf{1}_{k}\left(\delta_{k}^{k}\right)^{\mathrm{T}}\right)\right)\left(V_{j}^{c}-V_{i}^{c}\right)^{\mathrm{T}} \\
= & \left(I_{k^{i-1}} \otimes B_{k} \otimes I_{k^{j-i-1}} \otimes B_{k} \otimes I_{k^{n-j-1}} \otimes D_{k}^{\mathrm{T}} B_{k}\right)\left(V_{n}^{c}-V_{i}^{\mathrm{C}}\right)^{\mathrm{T}} \\
& -\left(I_{k^{i-1}} \otimes B_{k} \otimes I_{k^{j-i-1}} \otimes B_{k} \otimes I_{k^{n-j-1}} \otimes D_{k}^{\mathrm{T}} B_{k}\right)\left(V_{n}^{c}-V_{j}^{c}\right)^{\mathrm{T}} \\
& +\left(I_{k^{i-1}} \otimes B_{k} \otimes I_{k^{j-i-1}} \otimes B_{k} \otimes I_{k^{n-j-1}}+\mathbf{1}_{k}\left(\delta_{k}^{k}\right)^{\mathrm{T}}\right)\left(V_{j}^{c}-V_{i}^{c}\right)^{\mathrm{T}} \\
= & \left(I_{k^{j-1}} \otimes B_{k} \otimes I_{k^{n-j-1}} \otimes D_{k}^{\mathrm{T}}\right)\left(I_{k^{i-1}} \otimes B_{k} \otimes I_{k^{n-i-1}} \otimes B_{k}\right)\left(V_{n}^{c}-V_{i}^{c}\right)^{\mathrm{T}} \\
& -\left(I_{k^{i-1}} \otimes B_{k} \otimes I_{k^{n-i-1}} \otimes D_{k}^{\mathrm{T}}\right)\left(I_{k^{j-1}} \otimes B_{k} \otimes I_{k^{n-j-1}} \otimes B_{k}\right)\left(V_{n}^{c}-V_{j}^{c}\right)^{\mathrm{T}} \\
& -\left(I_{k^{n-2}} \otimes \mathbf{1}_{k}\right)\left(I_{k^{i-1}} \otimes B_{k} \otimes I_{k^{j-i-1}} \otimes B_{k} \otimes I_{k^{n-j-1}} \otimes\left(\delta_{k}^{k}\right)^{\mathrm{T}}\right)\left(V_{j}^{c}-V_{i}^{c}\right)^{\mathrm{T}} \\
= & 0 .
\end{aligned}
$$

(iii) $\Rightarrow$ (ii) Multiplying (75) on the left by $I_{k^{n-1}} \otimes\left(\delta_{k}^{k}\right)^{\mathrm{T}}$ yields (74).

(ii) $\Rightarrow$ (i) Since $T_{3} \tilde{b}=0$ is just (73), by Theorem 15 , we only need to check $S_{2} T_{2}=0$, i.e.

$$
M_{i+1, i} T_{2 i} \tilde{b}_{i}-\sum_{j=i+1}^{n-1} L_{i+1, j} T_{2 j} \tilde{b}_{j}=0
$$

for all $i=1,2, \cdots, n-2$. With simple calculations, we have

$$
\begin{aligned}
& L_{i+1, j} T_{2 j} \\
= & \left(I_{k^{i-1}} \otimes B_{k} \otimes D_{k^{n-i-1}}\left(I_{k^{j-i-1}} \otimes D_{k}^{\mathrm{T}} \otimes \mathbf{1}_{k^{n-j-1}}\left(\delta_{k^{n-j-1}}^{k^{n-j-1}}\right)^{\mathrm{T}}\right)\right) \\
& \quad \cdot\left(I_{k^{j-1}} \otimes B_{k} \otimes I_{k^{n-j-1}} \otimes\left(\delta_{k}^{k}\right)^{\mathrm{T}}\right) \\
= & \left(I_{k^{i-1}}(k-1) \otimes D_{k^{n-i-1}}\right)\left(I_{k^{j-2}}(k-1)^{\mathrm{T}} \otimes D_{k}^{\mathrm{T}} \otimes \mathbf{1}_{k^{n-j-1}}\left(\delta_{k^{n-j-1}}^{k^{n-j-1}}\right)^{\mathrm{T}}\right) \\
& \quad \cdot\left(I_{k^{i-1}} \otimes B_{k} \otimes I_{k^{j-i-1}} \otimes B_{k} \otimes I_{k^{n-j-1}} \otimes\left(\delta_{k}^{k}\right)^{\mathrm{T}}\right) .
\end{aligned}
$$

From (74) and (81), it follows that

$$
L_{i+1, j} T_{2 j} \tilde{b}_{j}=L_{i+1, j} T_{2 j} \tilde{b}_{i} .
$$

Therefore, by (82) and (58), we have

$$
\begin{aligned}
& M_{i+1, i} T_{2 i} \tilde{b}_{i}-\sum_{j=i+1}^{n-1} L_{i+1, j} T_{2 j} \tilde{b}_{j} \\
= & \left(M_{i+1, i} T_{2 i}-\sum_{j=i+1}^{n-1} L_{i+1, j} T_{2 j}\right) \tilde{b}_{i} \\
= & \left(\left(M_{i+1, i} \Phi_{i}-\sum_{j=i+1}^{n-1} L_{i+1, j} \Phi_{j}\right) \otimes\left(\delta_{k}^{k}\right)^{\mathrm{T}}\right) \tilde{b}_{i}=0 .
\end{aligned}
$$


Using the concept of multi-indexed matrix proposed by Cheng (2012), we can simplify (75). For details of multiindexed matrix, please refer Definition 1.1 and Definition 1.3 of Cheng (2012). Here, we only give an intuitive example. Given a 4-dimensional data

$$
X=\left\{x_{i_{1} i_{2} i_{3} i_{4}} \mid i_{1}=1,2 ; i_{2}=1,2,3 ; i_{3}=1,2 ; i_{4}=1,2,3,4\right\},
$$

we arrange $X$ into a matrix

$$
X_{23}^{14}=\left[\begin{array}{rrrrrr}
x_{11}^{11} & x_{12}^{11} & x_{21}^{11} & x_{22}^{11} & x_{31}^{11} & x_{32}^{11} \\
x_{11}^{12} & x_{12}^{12} & x_{21}^{12} & x_{22}^{12} & x_{31}^{12} & x_{32}^{12} \\
x_{11}^{13} & x_{12}^{13} & x_{21}^{13} & x_{22}^{13} & x_{31}^{13} & x_{32}^{13} \\
x_{11}^{14} & x_{12}^{14} & x_{21}^{14} & x_{22}^{14} & x_{31}^{14} & x_{32}^{14} \\
x_{11}^{21} & x_{12}^{21} & x_{21}^{21} & x_{22}^{21} & x_{31}^{21} & x_{32}^{21} \\
x_{11}^{22} & x_{12}^{22} & x_{21}^{22} & x_{22}^{22} & x_{31}^{22} & x_{32}^{22} \\
x_{11}^{23} & x_{12}^{23} & x_{21}^{23} & x_{22}^{23} & x_{31}^{23} & x_{32}^{23} \\
x_{11}^{24} & x_{12}^{24} & x_{21}^{24} & x_{22}^{24} & x_{31}^{24} & x_{32}^{24}
\end{array}\right],
$$

where $x_{i_{2} i_{3}}^{i_{1} i_{4}}=x_{i_{1} i_{2} i_{3} i_{4}}$, the multi-index of the rows of $X_{23}^{14}$ is $i_{1} i_{4}$ and the multi-index of the columns is $i_{2} i_{3}$. We usually say that $X_{23}^{14}$ is in the order of $\operatorname{id}\left(i_{1}, i_{4} ; 2,4\right) \times$ $\operatorname{id}\left(i_{2}, i_{3} ; 3,2\right)$.

Lemma 23 Suppose $Y=\left(A_{1} \otimes A_{2} \otimes \cdots \otimes A_{l}\right) X$, where $X$ and $Y$ be column vectors and $A_{i} \in \mathbb{R}^{m_{i} \times n_{i}}$ for each $i=1,2, \cdots, m$. Assume that the elements of $X$ and $Y$ are in the order of $\operatorname{id}\left(j_{1}, \cdots, j_{l} ; n_{1}, \cdots, n_{l}\right)$ and $\operatorname{id}\left(i_{1}, \cdots, i_{l} ; m_{1}, \cdots, m_{l}\right)$ respectively. Then

$Y_{r_{1} \cdots r_{l-t}}^{s_{1} \cdots s_{t}}=\left(A_{s_{1}} \otimes \cdots \otimes A_{s_{l}}\right) X_{r_{1} \cdots r_{l-t}}^{s_{1} \cdots s_{t}}\left(A_{r_{1}} \otimes \cdots \otimes A_{r_{l-t}}\right)^{\mathrm{T}}$,

where $\left\{s_{1}, \cdots, s_{t}, r_{1}, \cdots, r_{l-t}\right\}=\{1,2, \cdots, l\}$.

By Lemma 23 and (75) of Theorem 22, we get the following corollary.

Corollary 24 Consider finite game $\mathcal{G}=(\mathcal{N}, \mathcal{S}, \mathcal{C})$ described by Definition 1 with payoff functions shown in (2). Let $R^{j \rightarrow i}$ be the multi-dimensional data of the relative payoffs with respect to $j>i$, i.e. $R^{j \rightarrow i}=V_{j}^{c}-V_{i}^{c}$. Then $\mathcal{G}$ is potential if and only if

$$
\left(B_{k} \otimes B_{k}\right)\left(R^{j \rightarrow i}\right)_{12 \cdots \hat{i} \cdots \hat{j} \cdots n}^{i j}=0
$$

for all $1 \leq i<j \leq n$, where a caret is used to denote missing terms.

Remark 25 Similar to Theorem 15, (84) is equivalent to

$$
\left(H_{k} \otimes H_{k}\right)\left(R^{j \rightarrow i}\right)_{12 \cdots \hat{i} \cdots \hat{j} \cdots n}^{i j}=0,
$$

which is consistent with the result of Sandholm (2010). But our result is derived from the potential equation.
Thus we have established a connection between the result of Cheng (2014) and that of Sandholm (2010).

For players $i$ and $j$, arbitrarily given the strategies of the other players, the payoffs of $i$ and $j$ admit a bimatrix game, which is called a bi-matrix sub-game of $\mathcal{G}$. Obviously, the relative payoffs of each bi-matrix subgame are just lie in one column of some $\left(R^{j \rightarrow i}\right)_{12 \cdots \hat{i} \cdots \hat{j} \cdots n}^{i j}$. Therefore, we have the following corollary.

Corollary 26 A finite game $\mathcal{G}$ is potential if and only if every bi-matrix sub-game of $\mathcal{G}$ is potential.

\section{$5 \quad$ An example}

Example 27 Consider a finite game $\mathcal{G}$ with $n=3, k=$ 2 and payoff matrix $C=\left(c_{i_{1} i_{2} i_{3}}^{\mu}\right)$. Let

$$
R=\left[\begin{array}{llllllll}
r_{111}^{1} & r_{112}^{1} & r_{121}^{1} & r_{122}^{1} & r_{211}^{1} & r_{212}^{1} & r_{221}^{1} & r_{222}^{1} \\
r_{111}^{2} & r_{112}^{2} & r_{121}^{2} & r_{122}^{2} & r_{211}^{2} & r_{212}^{2} & r_{221}^{2} & r_{222}^{2}
\end{array}\right]
$$

where each $r_{i j k}^{\mu}=c_{i j k}^{3}-c_{i j k}^{\mu}$ for $\mu=1,2$. A computation shows that the coefficient matrix of (63) is

$$
\left[\begin{array}{rrrrrrrrrrrrrrrr}
0 & 1 & 0 & -1 & 0 & -1 & 0 & 1 & 0 & -1 & 0 & 1 & 0 & 1 & 0 & -1 \\
1 & -1 & 0 & 0 & -1 & 1 & 0 & 0 & 0 & 0 & 0 & 0 & 0 & 0 & 0 & 0 \\
0 & 0 & 1 & -1 & 0 & 0 & -1 & 1 & 0 & 0 & 0 & 0 & 0 & 0 & 0 & 0 \\
0 & 0 & 0 & 0 & 0 & 0 & 0 & 0 & 1 & -1 & -1 & 1 & 0 & 0 & 0 & 0 \\
0 & 0 & 0 & 0 & 0 & 0 & 0 & 0 & 0 & 0 & 0 & 0 & 1 & -1 & -1 & 1
\end{array}\right] .
$$

Thus, by Theorem 20, the game is a potential game if and only if

$$
\left\{\begin{array}{l}
r_{112}^{1}-r_{122}^{1}-r_{212}^{1}+r_{222}^{1}-r_{112}^{2}+r_{122}^{2}+r_{212}^{2}-r_{222}^{2}=0 \\
r_{111}^{1}-r_{112}^{1}-r_{211}^{1}+r_{212}^{1}=0 \\
r_{121}^{1}-r_{122}^{1}-r_{221}^{1}+r_{222}^{1}=0 \\
r_{111}^{2}-r_{112}^{2}-r_{211}^{2}+r_{212}^{2}=0 \\
r_{121}^{2}-r_{122}^{2}-r_{221}^{2}+r_{222}^{2}=0
\end{array}\right.
$$

which has the minimal number of equations for detecting whether $\mathcal{G}$ is potential.

\section{Conclusions}

For detecting whether a finite game is potential, new necessary and sufficient conditions have been obtained by investigating the potential equations. The number of the obtained verification equalities is minimal. The connections between the potential equations and the existing results on potential games have been revealed. It has 
been shown that a finite game is potential if and only if its every bi-matrix sub-game is potential. In the future work, we will use the potential equation to investigate near-potential games, networked game and so on.

\section{References}

Candogan O., Menache, I., Ozdaglar, A., \& Parrilo, P. A. (2011). Flows and Decompositions of Games: Harmonic and Potential Games. Mathematics of Operations Research, 36(3), 474-503.

Cheng, D., Qi, H., \& Zhao, Y. (2012). An introduction to semi-tensor product of matrices and its applications. Singapore: World Scientific.

Cheng, D. (2014). On finite potential games. Automatica, 50(7): 1793-1801.

Cheng, D., Xu, T., \& Qi, H. (2014). Evolutionarily stable strategy of networked evolutionary games. IEEE Transactions on Neural Networks and Learning Systems, 25(7), 1335-1345.

Cheng, D., Xu, T., He, F., \& Qi, H. (2014). On dynamics and Nash equilibriums of networked games, IEEE/CAA Journal of Automatica Sinica, 1(1), 1018.

Cheng, D., He, F., Qi, H., \& Xu, T. (2015). Modeling, analysis and control of networked evolutionary games. IEEE Transactions on Automatic Control, DOI: $10.1109 /$ TAC.2015.2404471.

Guo, P., Wang, Y., \& Li, H. (2013). Algebraic formulation and strategy optimization for a class of evolutionary networked games via semi-tensor method. Automatica, 49(11), 3384-3389.

Hino Y. (2011). An improved algorithm for detecting potential games. International Journal of Game Theory, 40(1), 199-205.

Horn, R. A., \& Johnson, C. R. Topics in matrix analysis. UK: Cambridge University Press, 1994.

Hwang, S.-H. \& Rey-Bellet L. (2011). Decompositions of two player games: potential, zero-sum, and stable games. arXiv:1106.3552v2.

Meyer, C. C., (2000). Matrix analysis and applied linear algebra. USA: Society for Industrial and Applied Mathematics.

Nash, J. (1951). Non-cooperative games. Annals of Mathematics, 54, 286-295.

Sandholm, W. H. (2010). Decompositions and potentials for normal form games. Games and Economic Behavior, 70, 446-456.

Monderer, D. \& Shapley L. S. (1996). Potential Games. Games and Economic Behavior, 14, 124-143.

Ui, T. (2000). A shapley value representation of potential games. Games and Economic Behavior, 31, 121135.

von Neumann, J. \& Morgenstern, O. (1953). Theory of Games and Economic Behavior. USA: Princeton University Press.

\section{Appendix: Proof of Lemma 18}

By the basic fact $D_{k}^{\mathrm{T}} B_{k}=I_{k}-\mathbf{1}_{k}\left(\delta_{k}^{k}\right)^{\mathrm{T}}$, we have

$$
\begin{aligned}
& -\sum_{j=1}^{n-1} N_{1 j} \Phi_{j} \\
= & \sum_{j=1}^{n-1} D_{k^{n-1}}\left(I_{k^{j-1}} \otimes D_{k}^{\mathrm{T}} B_{k} \otimes \mathbf{1}_{k^{n-j-1}}\left(\delta_{k^{n-j-1}}^{k^{n-j-1}}\right)^{\mathrm{T}}\right) \\
= & D_{k^{n-1}} \sum_{j=1}^{n-1} I_{k^{j-1}} \otimes\left(I_{k}-\mathbf{1}_{k}\left(\delta_{k}^{k}\right)^{\mathrm{T}}\right) \otimes \mathbf{1}_{k^{n-j-1}}\left(\delta_{k^{n-j-1}}^{k^{n-j-1}}\right)^{\mathrm{T}} \\
= & D_{k^{n-1}}\left(\sum_{j=1}^{n-1} I_{k^{j}} \otimes \mathbf{1}_{k^{n-j-1}}\left(\delta_{k^{n-j-1}}^{k^{n-j-1}}\right)^{\mathrm{T}} \sum_{j=1}^{n-1} I_{k^{j-1}} \otimes \mathbf{1}_{k^{n-j}}\left(\delta_{k^{n-j}}^{k^{n-j}}\right)^{\mathrm{T}}\right) \\
= & D_{k^{n-1}}\left(I_{k^{n-1}}-\mathbf{1}_{k^{n-1}}\left(\delta_{k^{n-1}}^{k^{n-1}}\right)^{\mathrm{T}}\right) \\
= & D_{k^{n-1}} D_{k^{n-1}}^{\mathrm{T}} B_{k^{n-1}} \\
= & B_{k^{n-1}}=\left[I_{k^{n-1}-1}-\mathbf{1}_{k^{n-1}-1}\right]
\end{aligned}
$$

For every $i=2,3, \cdots, n-1$, similarly, we have

$$
\begin{aligned}
& \quad-M_{i, i-1} \Phi_{i-1}-\sum_{j=i}^{n-1} L_{i j} \Phi_{j} \\
& =I_{k^{i-2}} \otimes B_{k} \otimes B_{k^{n-i}}-\sum_{j=i}^{n-1} I_{k^{i-2}} \otimes B_{k} \otimes\left(D_{k^{n-i}}\right. \\
& \left.\qquad \cdot\left(I_{k^{j-i}} \otimes D_{k}^{\mathrm{T}} B_{k} \otimes \mathbf{1}_{k^{n-j-1}}\left(\delta_{k^{n-j-1}}^{k^{n-j-1}}\right)^{\mathrm{T}}\right)\right) \\
& =I_{k^{i-2}} \otimes B_{k} \otimes B_{k^{n-i}}-\sum_{j=i}^{n-1} I_{k^{i-2}} \otimes B_{k} \otimes\left(D_{k^{n-i}}\right. \\
& \left.\quad \cdot\left(I_{k^{j-i}} \otimes\left(I_{k}-\mathbf{1}_{k}\left(\delta_{k}^{k}\right)^{\mathrm{T}}\right) \otimes \mathbf{1}_{k^{n-j-1}}\left(\delta_{k^{n-j-1}}^{k^{n-j-1}}\right)^{\mathrm{T}}\right)\right) \\
& =I_{k^{i-2}} \otimes B_{k} \otimes B_{k^{n-i}}-I_{k^{i-2}} \otimes B_{k} \otimes D_{k^{n-i}}\left(I_{k^{n-i}}-\mathbf{1}_{k^{n-1}}\left(\delta_{k^{n-i}}^{k^{n-i}}\right)^{\mathrm{T}}\right) \\
& =I_{k^{i-2}} \otimes B_{k} \otimes B_{k^{n-i}}-I_{k^{i-2}} \otimes B_{k} \otimes D_{k^{n-i}} D_{k^{n-i}}^{\mathrm{T}} B_{k^{n-i}} \\
& =0 .
\end{aligned}
$$

Moreover, by the fact that $B_{k} D_{k}^{\mathrm{T}}=I_{k-1}$ we have

$$
\begin{aligned}
M_{i, i-1} G_{i-1} & =\left(I_{k^{i-2}(k-1)} \otimes B_{k^{n-i}}\right)\left(I_{k^{i-2}(k-1)} \otimes D_{k^{n-i}}^{\mathrm{T}}\right) \\
& =I_{k^{i-2}(k-1)\left(k^{n-i}-1\right)} .
\end{aligned}
$$

From $D_{k} \delta_{k}^{k}=0$, it follows that

$$
\begin{aligned}
L_{i j} G_{j}= & -\left(I_{k^{i-2}} \otimes B_{k} \otimes N_{i j}\right)\left(I_{k^{j-1}}(k-1) \otimes D_{k^{n-j-1}}^{\mathrm{T}}\right) \\
= & -I_{k^{i-2}} \otimes B_{k} \otimes N_{i j}\left(I_{k^{j-i}(k-1)} \otimes D_{k^{n-j-1}}^{\mathrm{T}}\right) \\
= & -I_{k^{i-2}} \otimes B_{k} \otimes D_{k^{n-i}}\left(I_{k^{j-i}} \otimes D_{k}^{\mathrm{T}}\right. \\
& \left.\otimes \mathbf{1}_{k^{n-j-1}}\left(\delta_{k^{n-j-1}}^{k^{n-1}}\right)^{\mathrm{T}}\right)\left(I_{k^{j-i+1}} \otimes D_{k^{n-j-1}}^{\mathrm{T}}\right) \\
= & -I_{k^{i-2}} \otimes B_{k} \otimes D_{k^{n-i}}\left(I_{k^{j-i}} \otimes D_{k}^{\mathrm{T}}\right. \\
& \left.\otimes \mathbf{1}_{k^{n-j-1}}\left(D_{k^{n-j-1}} \delta_{k^{n-j-1}}^{k^{n-j-1}}\right)^{\mathrm{T}}\right) \\
= & 0 .
\end{aligned}
$$

From (86)-(89), we get (59). 